\title{
La caracterización de pacientes como herramienta útil para ofertar servicios profesionales personalizados en farmacia comunitaria
}

\author{
Ma José Martín Calero ${ }^{1,2}, \mathrm{M}^{a}$ José de la Matta Martín ${ }^{1,2}$, Fernando Marín Isorna ${ }^{3}$, Rosa Lorenzo Murillo², \\ Ana Luis Amado², Isabel Villegas Lama ${ }^{5}$
}

1. Doctora en Farmacia. Grupo de Investigación en Farmacoterapia y Atención Farmacéutica, CTS-528, Universidad de Sevilla. 2. Farmacéutica comunitaria en Sevilla. 3. Farmacéutico. Máster en Atención Farmacéutica y Farmacia Asistencial. Universidad de Sevilla. 5. Profesora Contratada Doctora del Departamento de Farmacología. Facultad de Farmacia, Universidad de Sevilla.

\section{PALABRAS CLAVE}

Farmacia comunitaria, cartera de servicios profesionales,

caracterización de pacientes

\section{ABREVIATURAS}

AF: Atención farmacéutica ANOVA: Análisis de la varianza ATC: Anatomical Therapeutic Chemical Classification System CC: circunferencia de cintura cHDL: colesterol unido a lipoproteínas de alta densidad cLDL: colesterol unido a lipoproteínas de baja densidad CV: cardiovascular

DM: diabetes mellitus

EAS: Encuesta Andaluza de Salud ENS: Encuesta Nacional de Salud

FC: farmacia comunitaria HTA: hipertensión arterial HBP: hiperplasia benigna de próstata IMC: índice de masa corporal INE: Instituto Nacional de Estadística

OMS: Organización Mundial de la Salud

PA: presión arterial

PAD: presión arterial diastólica PAS: presión arterial sistólica

RCV: riesgo cardiovascular

SFT: seguimiento

farmacoterapéutico

SPD: sistema personalizado de dosificación

$\chi^{2}$ : Chi-cuadrado

\section{KEYWORDS}

Community pharmacy, professional services, patients' characterization

\section{RESUMEN}

Introducción: El futuro inmediato de la farmacia requiere la incorporación de servicios profesionales orientados a optimizar la gestión personalizada del paciente.

Objetivos del trabajo: Determinar las características de los pacientes/usuarios registrados en la base de datos de una farmacia comunitaria con el fin de detectar sus necesidades sanitarias para establecer las pautas de intervención más adecuadas y eficaces.

Material y métodos: Se diseñó un cuestionario adaptado al ámbito de la farmacia comunitaria que facilitara el almacenamiento y tratamiento de los datos de los pacientes/usuarios seleccionados a través de entrevistas personales.

Resultados: En la muestra predominan los pacientes pluripatológicos $(80,5 \%)$ y polimedicados $(72,2 \%)$ de edad avanzada $(70,6 \pm 9,8$ años) con bajo nivel de estudios $(86,1 \%)$. Dislipemia $(63,9 \%)$, HTA $(61,1 \%)$, artrosis $(56,6 \%)$, enfermedades vasculares periféricas $(38,9 \%)$ y diabetes II $(30,6 \%)$ fueron las patologías más prevalentes. Aunque el 69,4\% realizaba ejercicio físico regularmente, el 81,8\% tenía exceso de peso y no realiza dietas adecuadas. El fármaco más consumido fue omeprazol $(97,0 \%)$ seguido de paracetamol $(50,0 \%)$, estatinas $(47,2 \%)$, benzodiacepinas, hidroclorotiazida y metformina.

Conclusión: La caracterización de pacientes a través de cuestionarios estructurados y consensuados pueden ayudar al farmacéutico a localizar los grupos más vulnerables a los que ofrecer servicios profesionales adaptados a sus necesidades y a elaborar programas de prevención y protocolos de actuación más eficientes.

Patient characterization as a useful tool for offering personalized professional services in the community pharmacy

\section{ABSTRACT}

Introduction: The immediate future of the pharmacy requires the incorporation of professional services aimed to optimizing personalized patient management.

Aims of the study: To determine the characteristics of patients registered in the database of a community pharmacy in order to detect their health needs, from these, establish guidelines most appropriate and effective intervention.

Material and methods: A questionnaire adapted to the field of community pharmacy was designed to facilitate the storage and processing of data of patients / users, who were registered in the pharmacy database, through personal interviews.

Results: Pluripatological (80.5\%) and polypharmacy patients $(72.2 \%)$, older $(70.6 \pm 9.8$ years) with low educational level $(86.1 \%)$ predominate in the study. Dyslipidemia (63.9\%), hypertension (61.1\%), arthritis (56.6\%), peripheral vascular disease (38.9\%) and diabetes II (30.6\%) were the most prevalent diseases. Although 69.4\% performed physical exercise regularly, almost 82\% were overweight and do not perform adequate diets. The most consumed drug was omeprazole (97.0\%), followed by acetaminophen (50.0\%), statins $(47.2 \%)$, benzodiazepines, hydrochlorothiazide and metformin.

Conclusion: The patients'characterization through structured and agreed questionnaires can help the pharmacist to locate the most vulnerable groups of the population studied in order to provide professional services adapted to their needs and develop prevention programs and more efficient protocols of action.

Declaración de autoria: MJMC e IVL participaron en el diseño del estudio, el desarrollo de la investigación, el análisis de los resultados, el tratamiento estadístico, la redacción del manuscrito y la revisión del contenido final. MJMM participó en el diseño del estudio, el desarrollo de la investigación, el análisis de los resultados y la revisión del contenido final. FMI participó en el desarrollo de la investigación, el análisis de los resultados, el tratamiento estadístico y la revisión del contenido final. RLM y ALM participaron en el desarrollo de la investigación.

Recibido: 2/10/2015

Aceptado: 28/11/2015

Disponible online: 1-12-2015
Financiación: La Fundación Farmacéutica Avenzoar ha concedido a este trabajo el Premio Reyes Malpica de investigación en 2014. También ha obtenido una de las cinco becas que la delegación andaluza de la Sociedad Española de Farmacia Familiar y Comunitaria, SEFAC, convocó para las mejores propuestas de proyectos de investigación procedentes de Andalucia presentadas al VI Congreso Nacional de Farmacéuticos Comunitarios, 2014. Un resumen del proyecto se presentó como comunicación en dicho congreso. Conflicto de intereses: Los autores declaran no existir conflicto de intereses en relación con el contenido del artículo.

Cite este artículo como: Martín MJ, Matta MJ, Marin F, Lorenzo R, Luis A, Villegas I. La caracterización de pacientes como herramienta útil para ofertar servicios profesionales personalizados en farmacia comunitaria. Farmacéuticos Comunitarios. 2015 Dec 01; 7(4):5-17. doi:10.5672/FC.2173-9218.(2015/Nol7).004.02

Autora para correspondencia: Mªsé Martin Calero (calero@us.es).

ISSN 1885-8619 @SEFAC (Sociedad Española de Farmacia Familiar y Comunitaria). Todos los derechos reservados. 


\section{Introducción}

Actualmente, las instituciones farmacéuticas y los propios farmacéuticos apuestan por ofertar nuevos servicios desde la farmacia comunitaria (FC) que potencien su actuación profesional (1-3). Su importancia radica no solo en ofrecer algo más que resulte beneficioso para los ciudadanos, sino que contribuyan realmente a mejorar su estado de salud por lo que deberían ir orientados a su integración en el sistema sanitario.

Los servicios profesionales que pueden ofertarse desde la FC son muy variados e incluyen desde la formulación magistral, o el seguimiento farmacoterapéutico (SFT) hasta la participación en programas de educación para la salud, colaboración en la adherencia a los tratamientos, intervención en procesos de deshabituación, farmacovigilancia, sistemas personalizados de dosificación (SPD) o cribado de enfermedades ocultas, entre otros muchos (1-3). Algunos servicios que requieren una titulación específica: análisis clínicos, ortopedia, óptica o elaboración de dietas, podrán proporcionarlos solo aquellas farmacias que cuenten con el personal titulado adecuado. Actualmente las instituciones farmacéuticas están proponiendo modelos retributivos que aún no se han implantado (4).

Estos procedimientos requieren una formación previa del farmacéutico que implica su acreditación para prestar el servicio y convenios de colaboración con otros profesionales sanitarios cuando se realizan de forma conjunta. Es esencial que el farmacéutico conozca las necesidades reales de sus pacientes a fin de poder ofertar lo que realmente le demandan y esto implica estudiar sus características sociodemográficas, sus patologías, hábitos de vida, tratamientos y una serie de parámetros bioquímicos $\mathrm{y}$ antropométricos. Los estudios de caracterización se justifican porque permiten implantar nuevos protocolos asistenciales o mejorar los servicios de salud ya existentes.

Nuestro grupo de trabajo ya estudió el perfil de pacientes de alto riesgo cardiovascular (RCV) atendidos en una unidad hospitalaria especializada. Tras evaluar sus características, se puso de manifiesto la necesidad de atenderlos en una consulta especialmente creada para ayudarles a ges- tionar su farmacoterapia al tiempo que se les aplicaba un protocolo de educación terapéutica (5). Dicha consulta quedó integrada en la unidad especializada de RCV y el médico especialista directamente derivaba a los pacientes a la farmacéutica que los atendía en ella.

Aunque este tipo de trabajos son frecuentes a nivel hospitalario $(6,7)$ no ocurre así en la FC. Hemos identificado el estudio EPIFARM (8) en 574 farmacias comunitarias españolas para identificar los perfiles de varones que solicitan medicación para la disfunción eréctil. García-Delgado et al (9), tras analizar el perfil de pacientes depresivos usuarios de la FC, pusieron de manifiesto las discrepancias entre los tratamientos y las guías de prescripción en esta patología, así como el grado de apoyo familiar y social que recibían. Más recientemente, Gómez Martínez et al (10) destacan el papel que pueden jugar los farmacéuticos en la caracterización, detección precoz y abordaje de pacientes con trastornos adaptativos usuarios de FC españolas. Es por tanto un tema novedoso que ofrece muchas posibilidades en la FC.

El objetivo principal del trabajo ha sido determinar las características de los pacientes registrados en la base de datos de una FC con el fin de detectar sus necesidades sanitarias para, a partir de estas, establecer las pautas de intervención más adecuadas y eficaces. Como objetivos específicos se plantean diseñar, a partir de cuestionarios ya existentes, uno adaptado al ámbito de la FC que permita el almacenamiento y tratamiento de los datos de los pacientes/usuarios; describir su perfil sociodemográfico, las patologías que padecen, los hábitos dietéticos e higiénico/sanitarios que siguen, así como registrar sus tratamientos.

\section{Material y métodos}

\section{Características del estudio}

Estudio observacional-descriptivo y transversal, realizado en una farmacia de Sevilla durante el periodo febrero-junio de 2013.

\section{Selección de los pacientes y procedimiento}

Los pacientes se seleccionaron de forma aleatoria entre los registrados como tales en la base de datos de la farmacia $(n=210)$. Cada paciente tiene asignado un número y se procedió a contactar con los múltiplos de 5. La invitación para participar se realizó vía telefónica o bien personalmente cuando acudían a la farmacia para retirar la medicación $(n=42)$.

Fueron incluidos en el estudio usuarios de ambos sexos mayores de edad, que recibieron información sobre el mismo, aceptaron participar y acudieron a la cita en el periodo indicado. Quedaron excluidos aquellos con alteraciones cognitivas que dificultaban su comunicación con el entrevistador, con discapacidad motora que dificultaban su desplazamiento hasta el lugar de la entrevista, que no aceptaron participar o bien, habiendo aceptado, no acudieron a la cita.

Todos los participantes firmaron el correspondiente consentimiento informado (Anexo I).

Los datos se obtuvieron a través de entrevista directa utilizando un cuestionario consensuado y estructurado, adaptado al entorno de la FC de otro empleado inicialmente en la caracterización de pacientes atendidos en una unidad especializada de RCV (5). Se realizaron algunos cambios valorados por un panel de expertos constituido por dos farmacéuticos comunitarios, una profesora del Departamento de Farmacología de la Universidad de Sevilla y dos miembros del Grupo de Investigación en Farmacoterapia y Atención Farmacéutica de esta Universidad (Anexo II).

Para la preparación de la entrevista se repasaron las fichas de atención farmacéutica (AF) registradas en el programa de gestión de la farmacia (Unycop Win v. 2013). El objetivo de esta fase previa fue prever la posible falta de respuesta por olvido o desconocimiento por parte de los pacientes entrevistados, aclarar dudas y, en algunos casos, orientar pero sin influir en la respuesta.

Las medidas de presión arterial (PA), peso, altura, indice de masa corporal (IMC) y circunferencia de la cintura (CC) se realizaron en el momento de la entrevista utilizando un tensiómetro Omron 705IT (PA), una balanza DINA Max 21 (IMC) y cinta métrica inextensible $(2 \mathrm{~m}$ de largo $\times 0,5 \mathrm{~cm}$ de ancho). También se pidió a los pacientes que llevaran a la cita la última analítica (últimos 
Tabla 1 Variables utilizadas en el proceso de caracterización

\begin{tabular}{|c|c|c|c|c|}
\hline \multirow[t]{2}{*}{ Variables } & \multicolumn{2}{|l|}{ Cualitativas (\%) } & \multicolumn{2}{|l|}{ Cuantitativas (Media \pm DE) } \\
\hline & Nombre & Tipo & Nombre & Tipo \\
\hline \multirow{4}{*}{ Socio-demográficas } & Sexo & Nominal & Edad & Razón \\
\hline & Nivel de formación & Nominal & Convivencia (número de personas) & Razón \\
\hline & Ocupación & Nominal & & \\
\hline & Estado civil & Nominal & & \\
\hline \multirow{2}{*}{$\begin{array}{l}\text { Relacionadas con } \\
\text { patologías }\end{array}$} & Patologías & Nominal & & \\
\hline & Patologias familiares & Nominal & & \\
\hline \multirow{15}{*}{ Hábitos saludables } & ¿Hace ejercicio? & Nominal & ¿Cuántas veces hace ejercicio? & Razón \\
\hline & Tipo de ejercicio & Nominal & ¿Cuánto tiempo de ejercicio? & Razón \\
\hline & ¿Camina habitualmente? & Nominal & ¿Durante cuánto tiempo? & Razón \\
\hline & ¿Algún sanitario le aconsejó bajar peso? & Nominal & ¿Cuánto le gustaría pesar? & Razón \\
\hline & ¿Está tratando de bajar peso? & Nominal & ¿Cuánto pesa? & Razón \\
\hline & ¿Suele tomar bebidas alcohólicas? & Nominal & ¿Cuántas veces al día? & Razón \\
\hline & ¿Fuma en la actualidad? & Nominal & Fumador ¿Cuántos cigarrillos fuma/dia? & Razón \\
\hline & ¿Última vez que fumó 1 cigarrillo/dia? & Nominal & & \\
\hline & $\begin{array}{l}\text { ¿Algún sanitario le aconsejó dejar el } \\
\text { tabaco? }\end{array}$ & Nominal & & \\
\hline & ¿Ha intentado dejar de fumar? & Nominal & & \\
\hline & ¿Piensa dejar de fumar? & Nominal & ¿En cuántos meses espera dejar de fumar? & Razón \\
\hline & ¿Hace algún tipo de dieta? & Nominal & Raciones de alimentos fritos (dia/semana) & Razón \\
\hline & ¿Fue instaurada por algún profesional? & Nominal & $\begin{array}{l}\text { Raciones de legumbres/ensaladas (dia/ } \\
\text { semana) }\end{array}$ & Razón \\
\hline & ¿Alguna restricción en su alimentación? & Nominal & Raciones de frutas (dia/semana) & Razón \\
\hline & ¿Añade sal a los alimentos cocinados? & Nominal & ¿Cuántas tazas de café toma al dia? & Razón \\
\hline \multirow{3}{*}{$\begin{array}{l}\text { Peso, IMC } \\
\text { y diámetro cintura }\end{array}$} & & & Altura medida $(\mathrm{m}) /$ Peso medido $(\mathrm{kg})$ & Razón \\
\hline & & & Índice de masa corporal & Razón \\
\hline & & & Contorno de cintura (cm) & Razón \\
\hline \multirow{3}{*}{$\begin{array}{l}\text { Parámetros } \\
\text { analíticos }\end{array}$} & ¿Controles de su glicemia capilar? & Nominal & & \\
\hline & ¿Controles de colesterol/TG capilar? & Nominal & & \\
\hline & ¿Controles de su tensión arterial? & Nominal & Medidas de tensión arterial & Razón \\
\hline
\end{tabular}

tres meses) para el control de los parámetros bioquímicos.

La clasificación de los fármacos incluidos en los tratamientos se ha realizado utilizando el sistema de codificación ATC (Anatomical Therapeutic Chemical Classification System).

Definición operacional y medida de las variables

Todas las variables registradas, con referencia a todas sus categorias, quedan reseñadas en los distintos apartados del Anexo II, pudiéndose distinguir varios grupos de las mismas para facilitar la mejor descripción de la muestra.

\section{Análisis estadístico} de los resultados

Las variables cuantitativas se expresan como media \pm desviación estándar, y las cualitativas como proporciones o porcentajes (frecuencias absolutas y relativas). El análisis estadístico realizado ha sido el test $t$ de Student y el análisis de la varianza
(ANOVA), seguido por el de Cohen, para las variables de tipo cuantitativo. Para las variables cualitativas se utilizó el test no paramétrico de Chí-cuadrado $\left(\chi^{2}\right)$ y análisis de residuos estandarizados corregidos ( $>2,7$ o 2,7). En los casos de comparaciones con una frecuencia inferior a 5 de una de las variables se utilizó el test exacto de Fischer. Se ha utilizado el programa SPSS (Statistical Package for Social Sciences) ${ }^{\circledR}$ versión 19 para Windows ${ }^{\circledR}$. Valores de $p<0,05$ se consideraron estadísticamente significativos. 


\section{Aspectos éticos}

Los procedimientos adoptados en esta investigación obedecen a los criterios de la ética en la investigación con los seres humanos conforme la Ley 14/1986, de 25 de abril, General de Sanidad (LGS).

La participación de los pacientes fue voluntaria tras haber recibido las oportunas explicaciones sobre el trabajo y haber firmado el consentimiento informado preceptivo. Para garantizar la confidencialidad se utilizó el código numérico que tienen asignados en la base de datos de la farmacia.

\section{Resultados y discusión \\ Datos sociodemográficos}

La muestra quedó reducida a 36 pacientes, ya que 6 declinaron la invitación a participar. La mayoría pluripatológicos $(80,5 \%)$ y polimedicados $(72,2 \%)$, de edad avanzada $(70,6 \pm 9,7$ años), siendo las mujeres algo más años) que los varones $(74,6 \pm 8,1$ años; rango 60-90 años). Solo el 33,3\% eran menores de 65 años y hubo ligero predominio del sexo femenino $(58,3 \%)$. Son personas de edad avanzada que, en sí misma, constituye un riesgo para múltiples patologías $(11,12)$.

La figura 1 refleja el nivel formativo de los participantes. En el epígrafe "estudios primarios" se incluyeron todas aquellas personas que supieran, al menos, leer, escribir y realizar opeEn cuanto a la ocupación desempeñada, sólo el 8,3\% ( $n=3$ mujeres) eran trabajadores activos. El resto $(91,7 \%)$ eran jubilados $(47,2 \%)$ o no habían realizado nunca trabajos remunerados (tareas del hogar, 44,5\%).

Todos estos condicionantes son importantes para orientar las entrejóvenes $(67,7 \pm 9$,8 años; rango 40-82 raciones básicas matemáticas $(86,1 \%)$.

vistas adaptándolas al nivel sociocultural del encuestado.

El 93,3\% de los hombres y el $81,0 \%$ de las mujeres estaban casados o con pareja $(86,1 \%$ del total, $n=31)$. Los restantes eran viudos $(n=5), 6,7 \%$ hombres y $19 \%$ mujeres. No obstante, el 94,4\% vivían acompañados ( $\mathrm{n}=34)$.

\section{Patologías prevalentes}

Las patologías de mayor incidencia entre la muestra estudiada se reflejan en la tabla 2. Una gran parte de los encuestados tenía los diagnósticos desde hacía 10 o más años: el 37,8\% de los que padecían dislipemias, el $45,5 \%$ de los hipertensos, la mayoría de los que padecen artrosis $(58,6 \%)$, más de la tercera parte de los diabéticos $(35,4 \%)$, la mitad de mujeres con antecedentes isquémicos $(50,0 \%)$ y el $(43,0 \%)$ de las que habían desarrollado osteoporosis. No se encontraron diferencias estadísticamente significativas entre el sexo del paciente y la aparición de la patología $(p>0,05)$.

Según la Encuesta Andaluza de Salud (EAS) 2011-2012 (13), las patologías más prevalentes en Andalucía fueron hipertensión arterial (HTA) $(18,7 \%)$, hipercolesterolemia $(12,3 \%)$ $\mathrm{y}$ artrosis o reumatismo (8,7\%). Cifras similares se registran a nivel nacional (Encuesta Nacional de Salud, ENS, 2011-12) (14), 18,7\%, 16,5\% y 19,4\%, respectivamente. Esta situación, aunque en porcentajes diferentes, concuerda bastante con los datos obtenidos entre nuestros pacientes. Hay que considerar que las encuestas citadas registran cifras de la población general mientras que en este trabajo analizamos personas mayores (70,6 $\pm 9,7$ años).

Por rangos de edad, la EAS indica prevalencias de hipercolesterolemia del 35,3\% y 34,3\% entre adultos de

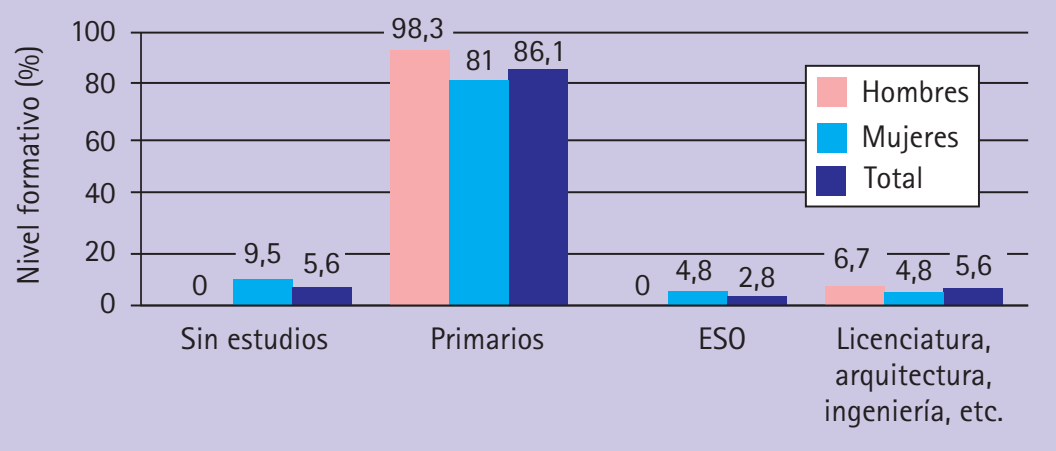

Figura 1 Nivel formativo de la muestra (distribución por sexos)
65-74 años y mayores (13) siendo estas cifras muy similares a nivel nacional (14). Se estima que en España el 20-25\% de los adultos de edades medias presentan cifras altas de colesterol y estas alcanzan hasta el 50-60\% entre los pacientes de RCV, valores parecidos a los países de nuestro entorno (15). El estudio ENRICA (16) señala un 51,1\% de adultos con problemas de hipercolesterolemia, un 44,9\% con cifras elevadas de cLDL y el 26\% con valores de cHDL bajos. Trabajos anteriores muestran cifras variables de prevalencia global de la dislipemia $(17,18)$, concluyéndose que los valores dependen de la edad de los participantes, del sexo, las áreas geográficas y las cifras a partir de las cuales se considera disfunción (colesterol total $>200$ o >250 mg/dl).

Algo similar ocurre respecto a la HTA. La EAS señala que la prevalencia es del 55,0\% entre 65-74 años y del 63,4\% en mayores de 75 años (13). Estas cifras son ligeramente más altas que a nivel nacional $(44,4 \%$ y $49,4 \%$ respectivamente) (14) y similares a las obtenidas para nuestra muestra $(61,1 \%)$. Otros estudios ofrecen cifras de $35-40 \%$ para adultos en edades medias y hasta el 68\% en mayores de 60 años $(18,19)$.

En los pacientes caracterizados, la incidencia de la artrosis y otras enfermedades reumáticas, 55,6\%, se asemeja a los porcentajes que refiere la ENS para adultos con edades avanzadas (46,7\% para el rango de 65-74 años y 58,1\% para mayores de 75) (14) y también coinciden con los datos descritos en otras fuentes (20). Sin embargo, se observan importantes diferencias a nivel autonómico (24,6\% y 35,7\%, respectivamente) para los mismos rangos de edad (13). Dado que nuestra población es andaluza cabría esperar un porcentaje menor de artríticos. Es posible que la selección de la muestra limitada al área de la farmacia pueda influir en estos resultados.

Las alteraciones vasculares periféricas (varices y hemorroides) afectaban al 38,9\% de los entrevistados, destacando las varices de miembros inferiores (tabla 2). Este porcentaje coincide con el descrito por otros autores que opinan que aproximadamente un tercio las padece de forma clínicamente significativa (21) y que la prevalencia es mayor en las mujeres, lo que también concuerda 
con los valores obtenidos entre la muestra.

El 38,5\% de los pacientes encuestados declaró padecer diabetes tipo II (DM II) (tabla 2) y la mayoría manifestó haber sido diagnosticados entre 1-5 años. Según la ENS, la prevalencia de esta enfermedad está en torno al 17,6-22\% entre pacientes mayores de 65 años (14) y la EAS sitúa ese porcentaje en el 27,6-30,8\% (13), cifras más parecidas a las registradas entre nuestros pacientes.

Los eventos isquémicos cardiovasculares (CV) (infarto agudo de miocardio (IAM), ictus, angina de pecho...) afectaban al 27,8\% de pacientes entrevistados (tabla 2) algo más a los varones que a las mujeres (60,0\% vs. $40,0 \%)$. Este resultado supera los porcentajes nacionales (14) y autonómico (13) para pacientes mayores $(12,0-20,1 \%$ y 9,6-20,0\%, respectivamente) y puede ser debido a la limitación de la muestra y al tipo de eventos CV registrados.

La hiperplasia benigna de próstata (HBP) es una patología exclusiva del hombre. El 50\% de los encuestados indican que la padecen entre 1-5 años. Se ha descrito que el 33\% de los hombres mayores de 50 años presenta adenoma bien constituido que llega al 95\% en los mayores de 80 años (22). Según la ENS (14) este porcentaje es menor, 29,5\%.

Aunque la osteoporosis se puede desarrollar en ambos sexos, solo uno de los hombres entrevistados manifestó estar en tratamiento preventivo. La refirieron el 19,4\% de las mujeres participantes y casi la mitad (42,9\%) desde hacía 10 o más años. Según la Guía de Buena Práctica Clínica en Osteoporosis (23), la prevalencia es superior en mujeres (4/1) y aumenta con la edad afectando casi al 60\% de las mayores de 80 años. A pesar de su elevada incidencia solo reciben tratamiento el 10\% de los afectados (23).

Los trastornos del ánimo son escasos entre nuestros pacientes (5,6\%) estando por debajo de la media nacional $(12,1 \%$ ansiedad y $11,1 \%$ depresión crónicas) (14) y andaluza (13,3-14,0\%) (13). La frustración por las limitaciones físicas y psíquicas y el dolor propio de sus patologías inciden negativamente en el psiquismo del anciano. Se ha descrito ampliamente que los mayores que viven solos suelen ser más proclives a padecer problemas de adaptación, tristeza, depresión y/o

Tabla 2 Patologías prevalentes en la muestra caracterizada (\%)

\begin{tabular}{|c|c|}
\hline Patologías & Porcentajes [H-M] \\
\hline 1. Dislipemias & $\begin{array}{c}63,9 \% \\
{[\text { H 53,3\%-M 42,9\%] }}\end{array}$ \\
\hline 2. Hipertensión arterial (HTA) & $\begin{array}{c}61,1 \% \\
{[\mathrm{H} 50,0 \%-\mathrm{M} \mathrm{50,0 \%}]}\end{array}$ \\
\hline 3. Artrosis & $\begin{array}{c}55,6 \% \\
{[\mathrm{H} 45,0 \%-\mathrm{M} \mathrm{55,0 \%}]}\end{array}$ \\
\hline 4. Hipertrofia Benigna de Próstata (HBP) ${ }^{1}$ & $53,3 \%$ \\
\hline $\begin{array}{l}\text { 5. Enfermedades vasculares periféricas: varices y } \\
\text { hemorroides }\end{array}$ & $\begin{array}{c}38,9 \% \\
{[\mathrm{H} 28,6 \%-\mathrm{M} 71,4 \%]}\end{array}$ \\
\hline 6. Diabetes tipo II & $\begin{array}{c}30,6 \% \\
{[\mathrm{H} 72,7 \%-\mathrm{M} 27,3 \%]}\end{array}$ \\
\hline $\begin{array}{l}\text { 7. Eventos isquémicos cardiovasculares: infarto agudo de } \\
\text { miocardio, ictus, angina de pecho... }\end{array}$ & $\begin{array}{c}27,8 \% \\
{[H 50,0 \%-M ~ 40,0 \%]}\end{array}$ \\
\hline 8. Osteoporosis & $\begin{array}{c}19,4 \% \\
{[\mathrm{H} 0,0 \%-\mathrm{M} 100,0 \%]}\end{array}$ \\
\hline 9. Arritmias Cardiacas & $11,1 \%$ \\
\hline 10. Artritis & $11,1 \%$ \\
\hline 11. Enfermedades oculares (glaucoma, cataratas, trombosis...) & $11,1 \%$ \\
\hline $\begin{array}{l}\text { 12. Digestivas: úlcera, hernia de hiato, enfermedad por } \\
\text { reflujo gastroesofágico... }\end{array}$ & $8,3 \%$ \\
\hline 13. Hipotiroidismo & $8,3 \%$ \\
\hline $\begin{array}{l}\text { 14. Intervenciones sobre el sistema óseo: menisco, columna, } \\
\text { cadera... }\end{array}$ & $8,3 \%$ \\
\hline 15. Insuficiencia cardiaca, soplo cardiaco... & $5,6 \%$ \\
\hline 16. Trastornos del equilibrio & $5,6 \%$ \\
\hline 17. Trastornos del ánimo: depresión, ansiedad, pánico... & $5,6 \%$ \\
\hline $\begin{array}{l}\text { 18. Alteraciones del metabolismo de purinas y pirimidinas: } \\
\text { hiperuricemias }\end{array}$ & $2,8 \%$ \\
\hline 19. Cefaleas & $2,8 \%$ \\
\hline 20. Fibromialgia & $2,8 \%$ \\
\hline 21. Hemocromatosis primaria & $2,8 \%$ \\
\hline 22. Hernia inguinal & $2,8 \%$ \\
\hline 23. Hipertiroidismo & $2,8 \%$ \\
\hline $\begin{array}{l}\text { 24. Otras enfermedades reumáticas como pericarditis, } \\
\text { miocarditis... }\end{array}$ & $2,8 \%$ \\
\hline 25. Queratosis actínica & $2,8 \%$ \\
\hline 26. Tuberculosis & $2,8 \%$ \\
\hline
\end{tabular}

1. Datos referidos a la polación masculina.

dejación que influyen negativamente en su estado de salud y en las posibilidades de éxito de las intervenciones planteadas (24). El hecho de que la mayor parte de los entrevistados vivan acompañados podría influir en los resultados obtenidos en esta muestra.

En cuanto a los antecedentes familiares, el 30,6\% de los encuestados refieren al padre $(11 / 36)$ como familiar más próximo que ha padecido la misma patología que ellos presentan, el $27,8 \%$ se refieren a la madre $(10 / 36)$ y el 19,4\% al hermano/a, siendo la HTA y las dislipemias las enfermedades más comúnmente citadas.

Hábitos higiénico-sanitarios Actividad física

El 69,4\% de los entrevistados hacía ejercicio con regularidad (25/36). 


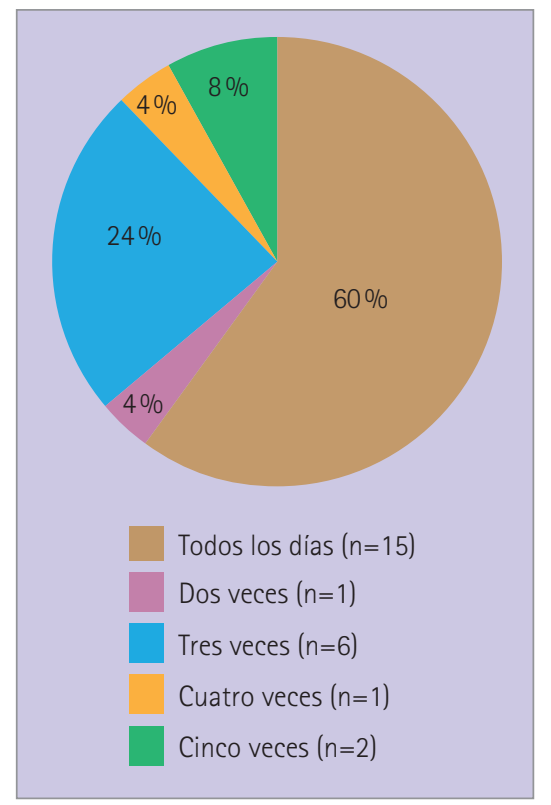

Figura 2 Actividad física de la muestra caracterizada (frecuencia semanal)

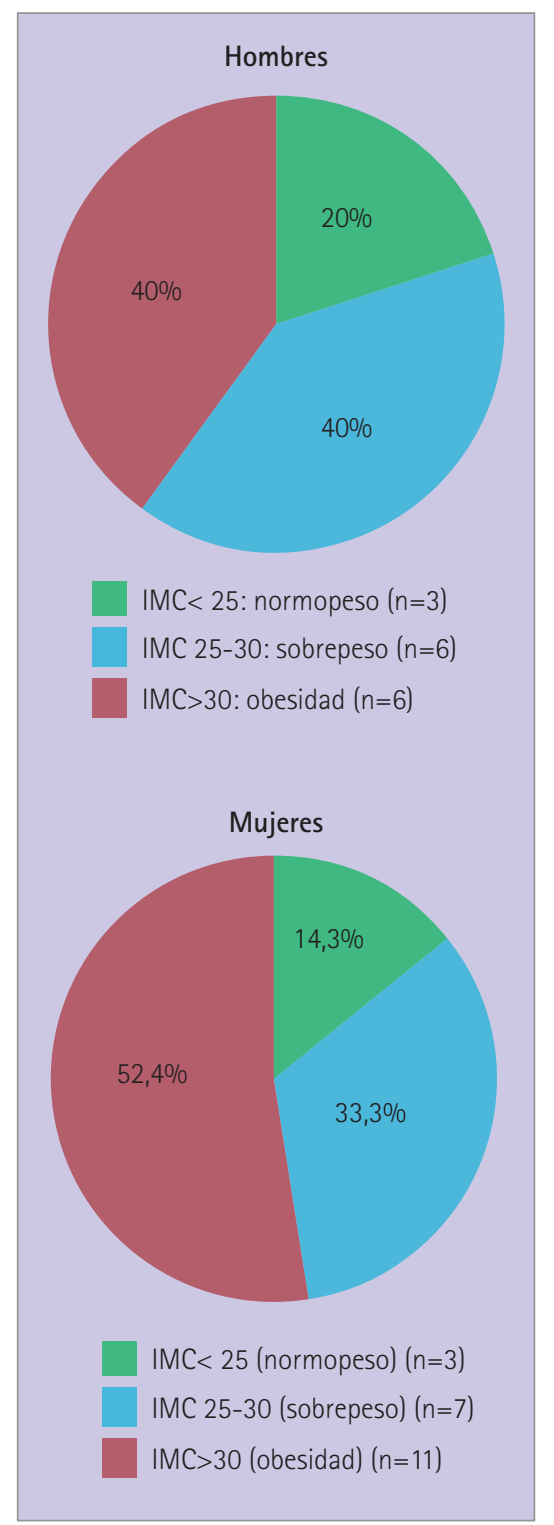

Figura 3 Índice de masa corporal de la muestra caracterizada
La principal actividad física desarrollada era caminar $(80,0 \%)$, seguida de la gimnasia de mantenimiento $(12,0 \%)$. El resto $(8,0 \%)$ practicaba múltiples actividades: pilates, natación... El 56,0\% de las personas activas eran mujeres (14/25) superando a los varones (11/25).

En la figura 2 se muestra la frecuencia de la actividad semanal realizada siendo muy variable la duración de las sesiones (45-120 minutos).

Cuando se practica regularmente, la actividad física es beneficiosa para reducir el riesgo de múltiples patologías, mejorar la salud músculoesquelética y contribuir al control del peso. Por el contrario, se estima que la inactividad es la causante del 21\%25\% de los cánceres de mama y colon, del $27 \%$ de los casos de diabetes y del $30 \%$ de las cardiopatías isquémicas (25). Para las personas mayores (>65 años) la OMS recomienda realizar al menos 150-300 minutos semanales de actividades aeróbicas (caminar a paso rápido, bicicleta, nadar...) en sesiones, como mínimo de 10 minutos.

\section{Consumo de tabaco y alcohol}

El $61,1 \%$ de los encuestados no eran consumidores habituales de bebidas alcohólicas (22/36), el 11,1\% reconoció haber bebido en alguna ocasión aunque actualmente lo había dejado (4/36) y solo el $27,8 \%$ era bebedor habitual (10/36, 60,0\% hombres). El consumo moderado de alcohol de 5-30 g/día, se considera beneficioso para la salud pues se asocia a una disminución en la morbimortalidad CV sin alterar el control glucémico (26). Deben evitar su ingesta aquellos enfermos que, como algunos de los entrevistados, toman antidepresivos, ansiolíticos o hipnóticos, lo que demuestra la necesidad de proporcionarles educación sanitaria para ayudarles en la gestión de su enfermedad.

En cuanto al consumo de tabaco, ninguno de los pacientes evaluados fumaba en el momento de la entrevista: el 63,9\% nunca lo había hecho con anterioridad (23/36, 20 mujeres) y el $36,1 \%$ lo había dejado (13/36). Más de la mitad de los exfumadores $(61,5 \%$ 8/13) habían sido aconsejados por el personal sanitario, al menos una vez, para que dejaran esta práctica. La relación entre las variables sexo/hábito de fumar resultó estadísticamente significativa ( $p<0,05)$.
El consumo de tabaco y alcohol así como el sedentarismo o el exceso de peso, son algunos de los factores de riesgo más importantes en el desarrollo de las principales enfermedades crónicas $(25,26)$. Gracias a las numerosas campañas realizadas cada vez se fuma menos (14) y la población se ha concienciado del peligro para la salud. Datos oficiales indican que, en Andalucía, la prevalencia de consumo cigarrillos/día se mantiene prácticamente estable, alrededor del 31\% desde 2000 (13) siendo los hombres los que más fuman $(39,4 \%$ frente al $23,1 \%$ de mujeres), preferentemente en el rango de edad de 25 a 44 años (43\%).

\section{Control del peso}

Índice de masa corporal. En la figura 3 se reflejan los porcentajes de IMC de los pacientes evaluados observándose el predominio del sobrepeso/ obesidad. Solo el 20,0\% de varones y el 14,3\% de mujeres presentaba normopeso (IMC<25) no encontrándose diferencias estadísticamente significativas entre ambos sexos $(\mathrm{p} \geq 0,05)$. Los porcentajes son algo diferentes a los que ofrece la ENS para el rango de edad de la muestra evaluada: obesos, $24,0 \%$ varones y $28,0 \%$ mujeres; sobrepeso, 53,4\% y 40,0\% respectivamente y normopeso, 22\% y 30,5\% (14). Respecto a la EAS casi el 60\% de los hombres tiene un peso superior al normal frente a las mujeres que alcanzan el 47\%, estando más afectados por el sobrepeso los rangos de edades entre 55 y 75 años (alrededor de 75\%) (13).

Circunferencia de la cintura. La media para esta variable en la muestra estudiada es 107,7 $\pm 11,7 \mathrm{~cm}$ (hombres $109,7 \pm 11,3 \mathrm{~cm}$, rango $88-130 \mathrm{y} \mathrm{mu}-$ jeres $106,2 \pm 12,1 \mathrm{~cm}$, rango $87-124$ ).

La OMS establece como límites deseables $102 \mathrm{~cm}$ para los varones y $88 \mathrm{~cm}$ para las mujeres. Solo 4 pacientes se ajustaban a estas medidas: $<102 \mathrm{~cm}(20,0 \%$ varones) y $<88 \mathrm{~cm}$ (4,8\% mujeres) superándolas la gran mayoría $(95,2 \% ; n=20)$. No se encontraron diferencias significativas entre ambos sexos.

La obesidad abdominal es importante porque está relacionada con el $\mathrm{RCV}$, incluyendo la prevalencia de diabetes y la resistencia a la insulina y en mujeres posmenopáusicas puede indicar la probabilidad de eventos con mayor acierto que el IMC (26-28) Por tanto, la asociación de un tratamiento 
Tabla 3 Distribución de los valores de presión arterial obtenida en los pacientes caracterizados. Clasificación de HTA propuesta por la Guía Europea de Práctica Clínica para el tratamiento de HTA (29)

\begin{tabular}{|c|c|c|c|c|c|}
\hline Categoría & PAS & & PAD & Hombres & Mujeres \\
\hline Óptima & $<120$ & y & $<80$ & - & $14,3 \%(n=3)$ \\
\hline Normal & $120-129$ & $y / 0$ & $80-84$ & $13,3 \%(n=2)$ & $19,1 \%(n=4)$ \\
\hline Normal alta & $130-139$ & y/o & $85-89$ & $20,0 \%(n=3)$ & $9,5 \%(n=2)$ \\
\hline Hipertensión de grado 1 & $140-159$ & y/o & $90-99$ & $6,7 \%(n=1)$ & $14,3 \%(n=3)$ \\
\hline Hipertensión de grado 2 & $160-179$ & y/o & $100-109$ & $6,7 \%(n=1)$ & - \\
\hline Hipertensión de grado 3 & $\geq 180$ & $y / 0$ & $\geq 110$ & - & - \\
\hline Hipertensión sistólica aislada & $\geq 140$ & $y / 0$ & $\leq 90$ & $53,3 \%(n=8)$ & $42,9 \%(n=9)$ \\
\hline
\end{tabular}

PAS: presión arterial sistólica; PAD: presión arterial diastólica.

farmacológico optimizado con hábitos de vida saludables ayuda a un mejor control de los factores de RCV.

\section{Control de la presión arterial (PA)}

Las medias correspondientes a la PA sistólica (PAS) y diastólica (PAD) de la muestra fueron de 141,3 $\pm 16,3$ y 79,3 $\pm 8,6 \mathrm{mmHg}$, respectivamente, siendo algo más elevada en los varones $(n=15$, PAS $146,5 \pm 11,9$ y PAD $79,9 \pm 8,4 \mathrm{mmHg}$ ) que en las mujeres $(\mathrm{n}=21$, PAS $137,6 \pm 18,2$ y PAD $78,9 \pm 8,9 \mathrm{mmHg}$ ) aunque sin diferencias significativas entre ambos grupos.

En la tabla 3 se observa que el porcentaje de hombres no hipertensos es inferior al de mujeres y abundan los casos de HTA sistólica aislada, característica de las personas de edad avanzada.

Es evidente que las cifras de PA aumentan con la edad. Según la ENS casi el 20\% de la población española ( $\geq 15$ años) tiene alta la PA y este porcentaje se eleva hasta el $44,4 \%$ en los mayores de 65 años (14). Por sexos, en menores de 64 años superan los varones hipertensos mientras que a edades más avanzadas predominan las mujeres. Teniendo en cuenta la edad de la muestra analizada en este trabajo, el porcentaje de varones con HTA es superior al referido en la ENS, sin embargo existe una gran concordancia respecto a las mujeres.

\section{Dieta y hábitos alimenticios}

La mayoría de los pacientes (91,7\%, n=33) realiza algún tipo de restricción alimentaria y en la figura 4 se refleja el porcentaje de alimentos más restringidos. El 50,0\% de las veces el régimen fue implantado por el personal de enfermería del centro de salud, un 10,0\% por el médico y el propio paciente se lo auto-impuso en un elevado porcentaje $(40,0 \%)$.

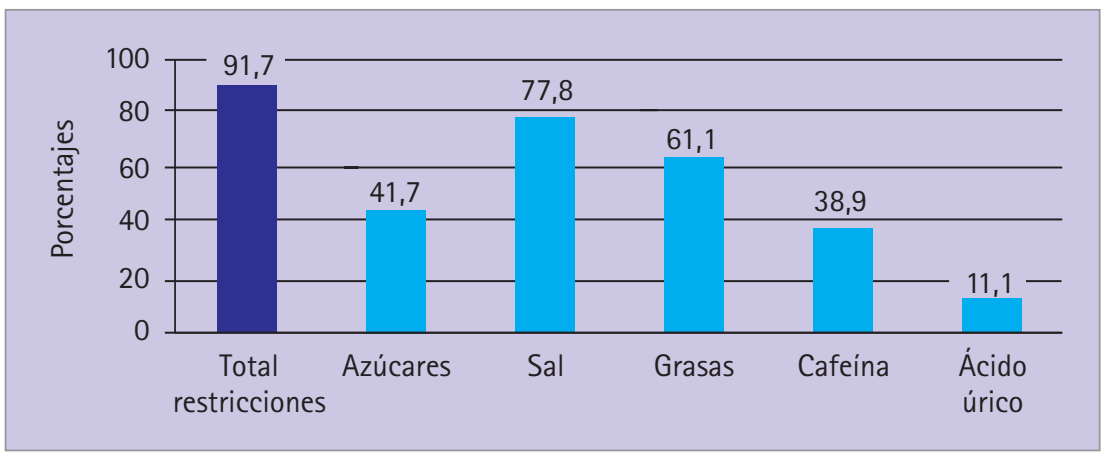

Figura 4 Restricciones alimentarias más habituales en la muestra caracterizada

El 72,2\% refirió tomar fritos solo 1-2 veces por semana y más de la mitad (58,3\%), dos o más veces al día legumbres/verduras/frutas. En cuanto al consumo de café, solo respondió afirmativamente el 16,7\% (6/36) y menos de 2 tazas/día. La mayor parte de la muestra $(77,8 \%$ [28/36]) lo tomaba descafeinado.

El 27,8\% de los pacientes (10/36) realizaba regímenes de adelgazamiento, preferentemente mujeres (7/10, $70,0 \%, p<0,05)$. Este dato es sensiblemente superior al porcentaje de personas que, según la ENS, hacen dieta en el rango de edad que estamos considerando (12,6\%) (14).

La alimentación es fundamental para prevenir muchas de las patologías referidas en este trabajo. El patrón de dieta mediterránea basada en la prevalencia de los hidratos de carbono sobre las grasas con abundante consumo de pescados, frutas y verduras, juega un papel importante en la consecución de estos beneficios terapéuticos $(30,31)$. Lamentablemente este modelo de alimentación se está perdiendo a favor del consumo de grasas saturadas (fast food). Una dieta equilibrada con un 50-60\% de hidratos de carbono (evitando los de azúcares de rápida metabolización tí- picos de dulces, bollería, refrescos...), un 10-15\% de proteínas y un 20-30\% de grasas (evitando las saturadas y el colesterol) contribuyen a optimizar la salud individual (30).

\section{Tratamientos utilizados}

Se muestran en la tabla 4. El fármaco más consumido ha sido omeprazol mayoritariamente en prevención de gastropatías $(97,0 \%)$ debido a la edad y los politratamientos de los pacientes. Le seguían paracetamol como analgésico $(50,0 \%)$, por delante de metamizol y antiinflamatorios no esteroideos; estatinas $(47,2 \%)$, principalmente simvastatina; benzodiacepinas, hidroclorotiazida y metformina. El 40,0\% de los varones con HBP utilizaba tamsulosina-dutasterida (Duodart $\left.{ }^{\circledR}\right)$. Estos datos concuerdan con los recomendados por diversas Guías y autores $(15,16,20,26,31)$ para pacientes en el rango de edad de la muestra caracterizada.

\section{Limitaciones del estudio}

Entre otras, se podría señalar la veracidad de la información suministrada por el paciente, que podría suponer un sesgo si las preguntas no las entiende bien o no las contesta 
Tabla 4 Tratamiento farmacológico de los pacientes caracterizados (distribución por grupo ATC, sexo, frecuencia y porcentaje)

\begin{tabular}{|c|c|c|c|c|}
\hline Grupos Terapéuticos & ATC & Sexo (n \%) & Frecuencia & Fármacos $\% *$ \\
\hline Antisecretores y procinéticos & $\begin{array}{l}\text { A02BC } \\
\text { A03FA }\end{array}$ & $\begin{array}{l}H: 14 ; 45,1 \\
M: 17 ; 27,3\end{array}$ & $n=31$ & 86,1 \\
\hline Analgésicos, antiinflamatorios & $\begin{array}{l}\text { N02B } \\
\text { N02A } \\
\text { N02C } \\
\text { M01A }\end{array}$ & $\begin{array}{l}H: 18 ; 69,2 \\
\text { M: } 8 ; 30,8\end{array}$ & $n=26$ & 72,20 \\
\hline $\begin{array}{l}\text { Antihipertensivos: diuréticos, alfa y beta-bloqueantes cardioselectivos, } \\
\text { calcioantagonistas, IECA, ARA II, y combinaciones }\end{array}$ & $\begin{array}{l}\text { C03AA } \\
\text { C03CA } \\
\text { C07AB } \\
\text { C08CA } \\
\text { C09AA } \\
\text { C09CA }\end{array}$ & $\begin{array}{l}H: 13 ; 56,5 \\
M: 10 ; 43,5\end{array}$ & $n=23$ & 64 \\
\hline Hipolipemiantes: estatinas, fenofibrato & $\begin{array}{l}\text { C10AA } \\
\text { C10AB }\end{array}$ & $\begin{array}{l}H: 13 ; 61,9 \\
M: 8 ; 30,1\end{array}$ & $n=21$ & 58,3 \\
\hline Hipnóticos, ansiolíticos, antidepresivos & $\begin{array}{l}\text { N05B } \\
\text { N05C } \\
\text { N06A }\end{array}$ & $\begin{array}{l}H: 10 ; 55,5 \\
M: 8 ; 44,5\end{array}$ & $n=18$ & 50 \\
\hline Antidiabéticos orales/insulinas & $\begin{array}{l}A 10 B A \\
A 10 B B \\
A 10 B D \\
A 10 B H \\
A 10 A\end{array}$ & $\begin{array}{l}H: 3 ; 27,3 \\
M: 8 ; 72,7\end{array}$ & $n=11$ & 38,5 \\
\hline $\begin{array}{l}\text { Prevención de ictus: antagonistas de la vitamina } K_{1} \text { antiagregantes } \\
\text { plaquetarios, }\end{array}$ & $\begin{array}{l}\text { B01AA } \\
\text { B01AC }\end{array}$ & $\begin{array}{l}H: 4 ; 40,0 \\
M: 6 ; 60,0\end{array}$ & $n=10$ & 28,0 \\
\hline $\begin{array}{l}\text { Tratamiento de la HBP: alfa-bloqueantes solos o asociados a inhibidores } \\
\text { de testosterona-5-alfa-reductasa }\end{array}$ & G04CA & $H: 8 ; 100,0$ & $n=8$ & $53,3^{* *}$ \\
\hline $\begin{array}{l}\text { Tratamiento de la osteoporosis: combinaciones de calcio, bifosfonatos, } \\
\text { ranelato de estroncio }\end{array}$ & $\begin{array}{l}\text { A12AX } \\
\text { M05BA } \\
\text { M05BX }\end{array}$ & $\begin{array}{l}H: 1 ; 20,0 \\
M: 4 ; 80,0\end{array}$ & $n=5$ & 14.0 \\
\hline Vasoprotectores & $\begin{array}{l}\text { C04AD } \\
\text { C05CA }\end{array}$ & $M: 3 ; 100,0$ & $n=3$ & 8,5 \\
\hline
\end{tabular}

* Porcentaje de fármacos utilizados en cada grupo terapéutico.

** Sobre el total de varones de la muestra caracterizada $(n=15)$.

verazmente. También el hecho de que todos sean usuarios habituales de la farmacia podría influir en los resultados. Esto se ha intentado minimizar ya que el entrevistador era una persona ajena al personal y el mismo para toda la muestra.

\section{Conclusiones}

El perfil encontrado ha sido el de un paciente mayor, con estudios primarios, pluripatológico y polimedicado, la mayoría con factores de RCV y/o en prevención secundaria, aunque también predominaban la artrosis y otras enfermedades reumáticas. Casi ninguno realizaba dietas adecuadas a su estado de salud a pesar de presentar sobrepeso o clara obesidad. Por tanto, estos pacientes precisarían optimizar su farmacoterapia y sería conveniente ofertarles servicios adecuados para contribuir a mejorar su estado de salud.

\section{Agradecimientos}

Los autores desean expresar su agradecimiento a la Fundación Farmacéutica Avenzoar y a la Sociedad Española de Farmacia Familiar y Comunitaria, SEFAC, por haber concedido a este trabajo, respectivamente, el Premio de investigación Reyes Malpica 2014 y una de las cinco becas de SEFAC Andalucía para las mejores propuestas de proyectos de investigación.

\section{Referencias bibliográficas}

1. Foro de Atención Farmacéutica en Farmacia Comunitaria. Cartera de servicios farmacéuticos en la farmacia comunitaria. Pharm Care Esp. 2012; 14(1): 31-32.

2. Baixauli Fernández VJ, Satué de Velasco E, Gil García MI, Roig Serna JC, Villasuso Cores B, Sáenz de Buruaga S. Propuesta de la Sociedad Española de Farmacia Comunitaria (SEFAC) sobre servicios profesionales farmacéuticos en farmacia comunitaria [Internet]. Documento SEFAC, 2013. [Acceso 12/6/15]. Disponible en: http://www.sefac.org/documentos $/ 34$ ? page $=1$

3. Colegio Oficial de Farmacéuticos de Sevilla-CECOFAR. La Cartera de Servicios de la farmacia sevillana: Una ventana abierta a una mayor calidad de vida. [Internet]. 2014. [Acceso 
19/6/15]. Disponible en: http://www. farmaceuticosdesevilla.es/cartera-de-servicios_aa342.html

4. Consejo General de Colegios Oficiales de Farmacéuticos. Servicios farmacéuticos: Definición y remuneración. [Internet]. CONGRAL, 2013, Madrid. [Acceso 12/6/15]. Disponible en: http://www.portalfarma.com/ Profesionales/organizacionfcolegial/ planfuturofarmaciacomunitaria/Documents/Servicios\%20Farmaceuticos_mayo\%202013.pdf

5. Marques Santana G, López Chozas JM, Pamies Andreu E, Pérez Guerrero C, Martin Calero MJ. Pharmacotherapeutical Profile in Patients With Dyslipemia Treated in a Especialized Unit in Vascular Risk. Meth Find Exper Clinl Pharmacol 2009; 31:66.

6. Ichikawa M, Takei Y, Hamasaki T, Kijima Y. Characterization of patients with angioscopically-detected in-stent mural thrombi. Circ J 2014; 79(1):8-590. doi:10.1253/circj.CJ-14-0639

7. Fliefel R, Tröltzsch M, Kühnisch J, Ehrenfeld M, Otto S. Treatment strategies and outcomes of bisphosphonate-related osteonecrosis of the jaw (BRONJ) with characterization of patients: a systematic review. Int J Oral Maxillofac Surg 2015. pii: S0901-5027(15)000582. doi. 1010.1016/ j.ijom.2015.01.026

8. Martín Morales A, Ibáñez J, Machuca M, Pol-Yanguas E, Schnetzler G, Pascual Renedo V. Estudio EPIFARM. Farmacéuticos comunitarios 2010; 2(4):140-145

9. García-Delgado Morente A, Machuca González M, Berenguer Froehner CB, Murillo Fernández MD, Martin Calero MJ, Cansino Calvo J, et al. Perfil del paciente ambulatorio usuario de fármacos antidepresivos. Grado de apoyo social y familiar. Pharm Care Esp 2009; 11(Suppl.):146.

10. Gómez Martínez J, López-Laguna Guerrero F, Ferragud Masià J, Abadías Guasch M. Perfil de los pacientes con trastorno adaptativo que acuden a las farmacias comunitarias españolas. Farmacéuticos Comunitarios 2014; 6(3):12-24. doi:10.5672/ FC.2173-9218.(2014/Vol6).003.03

11. Arai H, Ouchi Y, Yokode M, Ito H, Uematsu H, Eto F, et al. Toward the realization of a better aged society: messages from gerontology and geriatrics. Geriatr Gerontol Int. 2012; 12(1):16-22. doi: 10.1111/j.1447-0594.2011. 00776.x

12. Widmeier K. Respect your elders Special considerations for EMS res- ponse to geriatric patients. JEMS 2013;38(8):36-41.

13. Encuesta Andaluza de Salud (EAS) 20112012. Muestra de Adultos. [Internet]. Consejería de Igualdad, Salud y Políticas Sociales, 2013. [Acceso 9/6/15]. Disponible en: http://www.juntadeandalucia.es/salud/export/sites/csalud/galerias/documentos/c_3 c 1 1 vida_sana/ EAS_2011_2012_Adultos.pdf

14. Encuesta Nacional de Salud (ENS) 2011-2012. Instituto Nacional de Estadística. Ministerio de Sanidad, Servicios Sociales e Igualdad [Internet]. 2013. [Acceso 9/6/15]. Disponible en: http://www.ine.es/prensa/np770.pdf

15. Millán Núñez-Cortés J, Alegría E, Alvarez-Sala Walther L, Ascaso Gimilio J, Lahoz Rallo C, Mantilla Morató $\mathrm{T}$, et al. Documento Abordaje de la Dislipemia. Sociedad Española de Arteriosclerosis (parte III). Clin Invest Arterioscl. 2012; 24(2):102-107. doi:10.1016/j.arteri.2011.09.004.

16. Guallar-Castillon P, Gil-Montero M, León-Muñoz LM, Ibáñez A, Bayán-Bravo A, Ibáñez JM et al. Magnitud y manejo de la hipercolesterolemia en la población adulta de España, 2008-2010: Estudio ENRICA. Rev Esp Cardiol. 2012; 65: 51- 8. http://dx. doi.org/10.1016/j.recesp.2012.02.005

17. Vegazo 0, Banegas JR, Civeira F, Serrano Aisa PL, Jiménez FJ, Luengo E et al. Prevalencia de dislipemia en las consultas ambulatorias del sistema nacional de salud: Estudio HISPALIPID. Med Clin 2006; 127(9):331-334. http://dx.doi.org/10.1157/13092314

18. Gabriel R, Alonso M, Segura A, Tormo MJ, Artigao LM, Banegas JR, et al. Prevalencia, distribución y variabilidad geográfica de los factores de riesgo cardiovascular en España. Análisis agrupado de datos individuales de estudios epidemiológicos poblacionales: Estudio ERICE. Rev Esp Cardiol. 2008; 61(10):1030-40. http://dx.doi. org/ $10.1157 / 13126043$

19. De la Sierra A, Gorostidi M, Marín R, Redón J, Banegas JR, Armario P, et al. Evaluación y tratamiento de la hipertensión arterial en España. Documento de consenso. Med Clin 2008;131(3):104116. doi:10.1157/13124015

20. Benito-Ruiz P, Calvet Fontova J, Lisbona Pérez P, Martínez Peromingo J, Möller Parera I, Monfort Faure J et al. Guía de Buena Práctica Clínica en Geriatría (GBPCG). Sociedad Española de Geriatría y Gerontología, Sociedad Española de Reumatología. Madrid: Elsevier España; 2008
21. Andras A, Ferket B. Screening for peripheral arterial disease. Cochrane Database Syst Rev. 2014; 4:CD010835. doi:10.1002/14651858

22. Parsons JK. Benign Prostatic Hyperplasia and Male Lower Urinary Tract Symptoms: Epidemiology and Risk Factors. Curr Bladder Dysfunct Rep. 2010;5(4):212-218. doi:10.1007/s118 84-010-0067-2

23. Carbonell Abella C, Martin Jimenez J, Valdes y Llorca C. Guía de la Buena Práctica Clínica en Osteoporosis. Toquero de la Torre F y Rodríguez Sendín JJ (Eds.), Madrid: International Marketing \& Communication; 2008.

24. Dennis M, Kadri A, Coffey J. Depression in older people in the general hospital: a systematic review of screening instruments. Age Ageing. 2012 Mar;41(2):14854. doi:10.1093/ageing/afr169

25. Taylor D. Physical activity is medicine for older adults. Postgrad Med J. 2014;90(1059):26-32. doi:10.1136/ postgradmedj-2012-131366

26. Grupo de Trabajo de la Guía de Práctica Clínica sobre Diabetes tipo 2. Plan Nacional para el Sistema Nacional de Salud del Ministerio de Sanidad y Consumo. Madrid: Agencia de Evaluación de Tecnologías Sanitarias. OSTEBA 2006/08; 2008.

27. Walker GE, Marzullo P, Ricotti R, Bona G, Prodam F. The pathophysiology of abdominal adipose tissue depots in health and disease. Horm Mol Biol Clin Investig. 2014;19(1):57-74. doi:10.1515/hmbci-2014-0023

28. Garcia-Labbé D, Ruka E, Bertrand OF, Voisine P, Costerousse 0, Poirier P. Obesity and Coronary Artery Disease: Evaluation and Treatment. Can J Cardiol. 2015;31(2):184-194. doi:10.1016/j.cjca.2014.12.008

29. Grupo de Trabajo para el Tratamiento de la Hipertensión Arterial de la Sociedad Europea de Hipertensión (ESH) y de la Sociedad Europea de Cardiología (ESC). Rev Esp Cardiol. 2007;60(9):968.e1-e94

30. Widmer RJ, Flammer AJ, Lerman LO, Lerman A. The Mediterranean Diet, its Components, and Cardiovascular Disease. Am J Med. 2015;128(3):229-238. doi:10.1016/j.amjmed.2014.10.014

31. Medlock S, Eslami S, Askari M, Taherzadeh Z, Opondo D, de Rooij SE et al. Co-prescription of gastroprotective agents and their efficacy in elderly patients taking nonsteroidal anti-inflammatory drugs: a systematic review of observational studies. Clin Gastroenterol Hepatol. 2013 0ct;11(10):1259-1269. e10. doi:10.1016/j.cgh.2013.05.034 


\section{ANEXO I. Consentimiento informado con cesión de datos}

D. /Dña con DNI. fecha de nacimiento sexo número de la Seguridad Social y con dirección en

El abajo firmante queda informado de forma precisa e inequivoca y autoriza expresamente a la Farmacia cuyos titulares son .. para recabar y tratar en un fichero del que es responsable los datos que se solicitan en el presente formulario. Asimismo, autorizo la cesión de mis datos personales recogidos en este establecimiento al Real e llustre Colegio Oficial de Farmacéuticos de Sevilla, sita en c/ Alfonso XII, 51 de Sevilla, cuya actividad es la ordenación del ejercicio de los profesionales farmacéuticos de la provincia, la representación exclusiva de las mismas y la defensa de los intereses profesionales de sus colegiados. La finalidad exclusiva de la cesión de datos es centralizar la información farmacoterapéutica sobre mi persona y que siempre con mi consentimiento expreso, puedan ser consultados durante el proceso de asistencia sanitaria por cualquier Oficina de Farmacia de la provincia de Sevilla. Le informamos que sus datos van a ser utilizados exclusivamente con la finalidad de prestarle un servicio de atención farmacéutica personalizado, esto es, facilitarle las siguientes funciones:

- Entregar el medicamento y/o el producto sanitario en condiciones óptimas y de acuerdo a la normativa legal vigente.

- Proteger al paciente frente a la posible aparición de problemas relacionados con los medicamentos.

- Indicar al paciente la actitud más adecuada para resolver su problema de salud, y en su caso seleccionar un medicamento.

- Resolver las dudas planteadas por el usuario o las carencias de información detectadas por el farmacéutico.

- Proteger al paciente frente a la posible aparición de problemas relacionados con los medicamentos.

- Evaluar si el problema de salud es precisamente consecuencia de un problema relacionado con un medicamento.

- Buscar la obtención de la máxima efectividad de los tratamientos farmacológicos.

- Minimizar los riesgos asociados al uso de los medicamentos y, por tanto, mejorar la seguridad de la farmacoterapia.

- Contribuir a la racionalización del uso de los medicamentos como principal herramienta terapéutica de nuestra sociedad.

- Mejorar la calidad de vida de los pacientes.

- Cualquier otra para las que se cuente con habilitación profesional

Datos que van a ser recabados al paciente:

- Antecedentes personales:

- dietas alimentarias especiales

- tabaquismo

- consumo de bebidas alcohólicas

- consumo de bebidas con cafeína

- historial de reacciones de hipersensibilidad

- historial de intolerancias

Enfermedades diagnosticadas y vacunas administradas.

- Historial de embarazo y lactancia.

- Historial farmacológico.

- Historial de acontecimientos adversos.

- Otros datos sanitarios de interés.

La información que se le solicita es facultativa, si bien, de no facilitarla no se le podrá prestar el servicio. Usted queda informado de que el anterior consentimiento es revocable y de que podrá ejercer sus derechos de acceso, rectificación, cancelación y oposición de conformidad con lo dispuesto en la legislación vigente en materia de protección de datos dirigiéndose por escrito a este establecimiento sanitario.

Fdo.: 


\title{
ANEXO II. Cuestionario de caracterización de la muestra
}

Fecha:

- Hora en que inicio este cuestionario:

h....

- Registrar la hora en que ha terminado este cuestionario: m

Duración de la entrevista: minutos

Farmacéutico:

\section{DATOS DEL PACIENTE}

Hombre $\square$

\author{
Mujer
}

Fecha de nacimiento:

Nivel de formación:

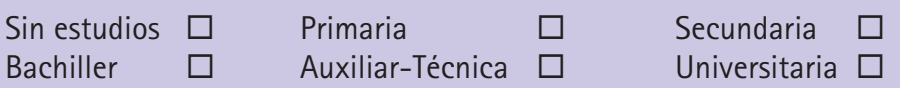

\section{Ocupación:}

Estado civil:

Soltero $\square \quad$ Casado (con pareja) $\square \quad$ Separado o divorciado $\square \quad$ Viudo $\square$

\section{PATOLOGÍAS}

Enfermedades que reconoce el paciente SI, si reconoce, o NO, si no reconoce.

\begin{tabular}{|l|l|l|l|l|l|}
\hline Enfermedad o afección & \multicolumn{4}{l|}{ Desde cuando conoce que la padece } \\
\hline Hipertensión arterial & $<6$ meses & $6-12$ meses & $1-5$ años & $5-10$ años & $>10$ años \\
\hline Dislipemia & $<6$ meses & $6-12$ meses & $1-5$ años & $5-10$ años & $>10$ años \\
\hline Diabetes tipo 2 & $<6$ meses & $6-12$ meses & $1-5$ años & $5-10$ años & $>10$ años \\
\hline Algún evento CV (IAM, angina de pecho, ictus...) & $<6$ meses & $6-12$ meses & $1-5$ años & $5-10$ años & $>10$ años \\
\hline Arritmias cardiacas & $<6$ meses & $6-12$ meses & $1-5$ años & $5-10$ años & $>10$ años \\
\hline Insuficiencia cardiaca & $<6$ meses & $6-12$ meses & $1-5$ años & $5-10$ años & $>10$ años \\
\hline Enfermedad arterial periférica & $<6$ meses & $6-12$ meses & $1-5$ años & $5-10$ años & $>10$ años \\
\hline Artritis & $<6$ meses & $6-12$ meses & $1-5$ años & $5-10$ años & $>10$ años \\
\hline Artrosis & $<6$ meses & $6-12$ meses & $1-5$ años & $5-10$ años & $>10$ años \\
\hline Otras enfermedades reumáticas & $<6$ meses & $6-12$ meses & $1-5$ años & $5-10$ años & $>10$ años \\
\hline Osteoporosis & $<6$ meses & $6-12$ meses & $1-5$ años & $5-10$ años & $>10$ años \\
\hline HBP & $<6$ meses & $6-12$ meses & $1-5$ años & $5-10$ años & $>10$ años \\
\hline & $<6$ meses & $6-12$ meses & $1-5$ años & $5-10$ años & $>10$ años \\
\hline & $<6$ meses & $6-12$ meses & $1-5$ años & $5-10$ años & $>10$ años \\
\hline & $<6$ meses & $6-12$ meses & $1-5$ años & $5-10$ años & $>10$ años \\
\hline
\end{tabular}

¿Tiene otras patologías? $\quad$ NO $\square \quad$ SI $\square$

Registrar otras patologías que el paciente reconoce que sufre:

¿ALGÚN FAMILIAR padece o ha padecido la enfermedad que usted tiene?

\begin{tabular}{|l|l|l|l|}
\hline Padre & & \\
\hline Madre & & \\
\hline Hermano/Hermana & & \\
\hline Hijo/Hija & & \\
\hline
\end{tabular}

Teléfono:

Correo electrónico: 


\section{HÁBITOS HIGIÉNICO-SANITARIOS}

\begin{tabular}{|l|l|}
\hline Seguro de Salud (Cobertura): & \\
\hline Centro de Salud: & Médico de Cabecera (tel) \\
\hline Clínica - Hospital & Médico especialista (tel) \\
\hline
\end{tabular}

\section{ACTIVIDAD FÍSICA}

¿Hace ejercicio físico regularmente? (Ejemplo: ir al gimnasio con horario marcado) No $\square \quad$ Sí

Si sí. ¿Qué ejercicio físico?

Si sí, ¿Cuántas veces por semana?

¿Cuántos días camina por semana? Días por semana ¿Cuánto tiempo emplea caminando? Horas:

Minutos: No camina

\section{CONSUMO DE ALCOHOL}

¿Suele beber cerveza o vino? No (He dejado de beber) $\square$

Si $\square \quad$ No consumo alcohol

\section{Si sí:}

¿Suele beber en las comidas?

¿Todos los días? ¿Cuántas veces?

¿Los fines de semana?

¿Qué bebidas consume?

\section{CONSUMO DE TABACO}

¿Actualmente fuma?
1. No (he dejado de fumar)

¿Cuándo fue la última vez que fumó por lo menos un cigarrillo diariamente?
1. Menos de 1 mes $\square$
2. Entre 1 y 6 meses $\square$
3. Entre 6 y 12 meses
4. Un año y más
5. No recuerdo/No estoy seguro .. años.

\section{Fumador}

¿Cuántos cigarrillos fuma al día?
0-1 paquete/diario CIGARRILLOS

¿Algún profesional de la salud le ha aconsejado que deje de fumar?
1. Sí, en alguna (s) ocasión (es) $\square$
4. No
2. Si, siempre me aconsejó
5. No estoy seguro

3. De qué profesional recibió el consejo?

¿Ha intentado alguna vez dejar de fumar? No $\square$

Si sí: ¿Cuantos veces ha intentado dejar de fumar?:

¿Piensa dejar de fumar?
1. No $\square$ 2. Sí $\square$
3. No recuerdo/No estoy seguro

¿En cuántos meses a partir de ahora espera conseguirlo? meses.

\section{CONTROL DE PESO}

¿Alguna vez un médico, enfermero u otro profesional sanitario le ha dicho que tiene sobrepeso, que está obeso o que pesa más de lo que debiera?

No $\square \quad$ Sí

¿Está usted en estos momentos tratando de bajar o perder peso? No

¿Cuánto cree usted que debería pesar?: Peso en $\mathrm{kg}$

¿Cuánto le gustaría pesar?: Peso en $\mathrm{kg}$

No estoy seguro No estoy seguro 
Cuánto mide?:

Medirlo:

m. Cuánto pesa?:

Pesarlo: $\mathrm{kg}$.

Según su peso, usted se considera que tiene o está:
a. Peso bajo
b. Peso normal
c. Sobrepeso
d. Obeso
e. No sabe

IMC

Circunferencia cintura CC

5. DIETAS Y HÁBITOS ALIMENTARIOS

¿Hace algún tipo de dieta? $\quad$ No $\square \quad$ Sí

¿Si Sí, la dieta fue instituida por un profesional de salud? $\quad$ No $\square \quad$ Si $\square$ ¿Qué profesional?

Médico $\square \quad$ Farmacéutico $\square \quad$ Enfermero $\square \quad$ Dietista

Otro ¿Cuál?

¿Hace alguna restricción en su alimentación?

No $\square \quad$ Sí $\square$

\section{¿Si sí, Cual?}

Restricción de hidratos de carbono (patata, arroz, etc.) No Sí

$\begin{array}{lll}\text { Restricción de azúcar (dosis) } & \text { No } \square & \text { Si } \square \\ \text { Restricción de sal } & \text { No } \square & \text { Si } \square \\ \text { Restricción de grasa } & \text { No } \square & \text { Si } \square \\ \text { Restricción de cafeína } & \text { No } \square & \text { Si } \square\end{array}$

¿Cuántas tazas de café toma?

$\square<2$ tazas por día $\square$ 2-6 tazas por día

¿Toma descafeinado?

$\square>6$ tazas por día

¿Le agrega o le pone sal a los alimentos una vez que están cocinados?

1. No, nunca

2. Si, cuando noto que le falta

3. Sí, siempre o casi siempre

¿Cuantas raciones cree que debería consumir?

\begin{tabular}{|l|l|l|l|l|l|l|}
\hline Alimentos fritos & 1 & 2 & 3 & 4 & 5 & / semana \\
\hline Legumbres/ensaladas & 1 & 2 & 3 & 4 & 5 & / dia o / semana \\
\hline Frutas & 1 & 2 & 3 & 4 & 5 & / día \\
\hline
\end{tabular}

\section{REGISTRO DE PARÁMETROS ANALITICOS}

Anotar los valores que presente el paciente del último control (máximo 6 meses) Determinar PA

Determinar glicemia capilar

Determinar otros parámetros bioquímicos vía seca (si posible)

\section{FARMACOTERAPIA DEL PACIENTE}

\begin{tabular}{|c|c|c|c|c|c|c|}
\hline $\begin{array}{c}\text { Principio } \\
\text { activo }\end{array}$ & $\begin{array}{c}\text { Grupo } \\
\text { Terapéutico }\end{array}$ & Indicación & Dosis & $\begin{array}{c}\text { Pauta } \\
\text { posológica }\end{array}$ & $\begin{array}{c}\text { Fecha de inicio } \\
\text { del tratamiento }\end{array}$ & $\begin{array}{c}\text { Fecha de finalización } \\
\text { del tratamiento }\end{array}$ \\
\hline & & & & & \\
\hline & & & & & \\
\hline & & & & & \\
\hline
\end{tabular}

